\title{
Force Control and
}

\section{Nonlinear Master-Slave}

Force Profile to Manage an Admittance Type Multi-Fingered Haptic User Interface

\section{International Symposium on Resilient Control Systems (ISRCS) 2012}

\author{
Anthony L. Crawford
}

The INL is a

U.S. Department of Energy

National Laboratory

operated by

Battelle Energy Alliance

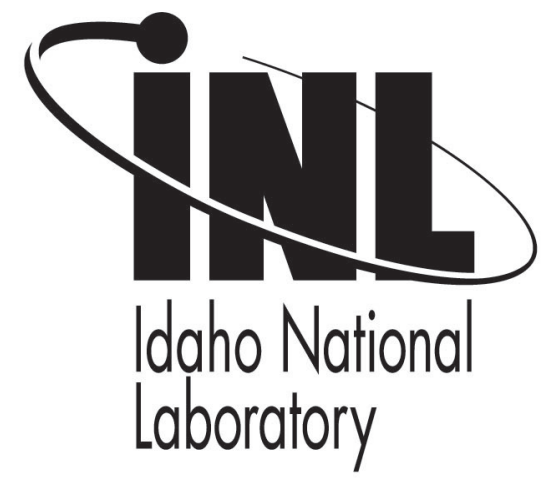

\section{August 2012}

This is a preprint of a paper intended for publication in a journal or proceedings. Since changes may be made before publication, this preprint should not be cited or reproduced without permission of the author. This document was prepared as an account of work sponsored by an agency of the United States Government. Neither the United States Government nor any agency thereof, or any of their employees, makes any warranty, expressed or implied, or assumes any legal liability or responsibility for any third party's use, or the results of such use, of any information, apparatus, product or process disclosed in this report, or represents that its use by such third party would not infringe privately owned rights. The views expressed in this paper are not necessarily those of the United States Government or the sponsoring agency. 


\title{
Force Control and Nonlinear Master-Slave Force Profile to Manage an Admittance Type Multi- Fingered Haptic User Interface
}

\author{
Anthony L. Crawford \\ Applied Engineering Department \\ Idaho National Laboratory \\ Idaho Falls, ID 83415 \\ Anthony.Crawford@inl.gov
}

\begin{abstract}
Natural movements and force feedback are important elements in using teleoperated equipment if complex and speedy manipulation tasks are to be accomplished in remote and/or hazardous environments, such as hot cells, glove boxes, decommissioning, explosive disarmament, and space to name a few. In order to achieve this end the research presented in this paper has developed an admittance-type exoskeleton like multifingered haptic hand user interface that secures the user's palm and provides 3-dimensional force feedback to the user's fingertips. Atypical to conventional haptic hand user interfaces that limit themselves to integrating the human hand's characteristics just into the system's mechanical design, this system also perpetuates that inspiration into the designed user interface's controller.

This is achieved by manifesting the property differences of manipulation and grasping activities as they pertain to the resilient human hand into a nonlinear master-slave force relationship. The results presented in this paper show that the admittance-type system has sufficient bandwidth such that it appears nearly transparent to the user when in free motion. Also, when executing a manipulation or grasping task, increased performance is achieved using the nonlinear force relationship compared to the traditional linear scaling techniques implemented in the vast majority of systems.
\end{abstract}

Keywords - force control, haptics, hand, robotic force scaling

\section{INTRODUCTION}

Teleoperation systems have been utilized extensively to handle objects in remote and/or hazardous environments with the most utilized design being patented in 1949 [1]. Some of the more recent, widely reported events where teleoperation systems have been used include reconnaissance in Japan's Fukishima nuclear power plant and explosive disarmament in Iraq and Afghanistan. Although these robots have been an invaluable resource in these applications they also have further displayed some of their shortcomings. A particular shortcoming includes their overly simple end-effector, whose lack of resiliency in design, feedback, and control even makes the act of operating a door knob, a task the human hand accomplishes with relative ease, difficult. In response to this circumstance this research is developing a haptic robotic hand capable of effectively operating in hazardous environments and a corresponding haptic hand user interface.
Although implementation of haptic robotic hands in hazardous environments has been limited, primarily due to difficulties associated with maintenance and the radiation intolerance of many electronics, the field of haptic hand user interfaces (HHUIs) that would control such a device has been extensively studied. The HHUIs that have been developed vary greatly in their interaction method with the human hand (e.g. thimbles, gloves, sensing surfaces), implemented actuators (e.g. DC motors, Shape memory alloys, ultrasonic, hydraulic), and kinematic structures (e.g. linkages, direct drive, gear driven, cable driven). However, a HHUI that has sufficient degrees of freedom (DOF) and bandwidth to accommodate dexterous/quick manipulation tasks AND sufficient strength to appropriately complement grasping tasks has not been found in the literature. This can be attributed to the difficulty associated with integrating the large number of sufficiently strong/quick actuators needed to complement all of the user's DOFs into the compact workspace around the user's hand and allow full movement of the hand and wrist.

In general, HHUIs and general haptic devices consist of two types of structures, these include impedance-type [3] and admittance-type [2][5]. The impedance-type devices, which are much more predominant, implement backdrivable actuators and position sensing to communicate a force back to the user (impedance control [9]). Their predominance is due to their reduced cost and simplicity at a consequence of a bulkier form and generally smaller force generating capacities. The admittance-type devices can be much more compact with the same strength capacities as that of impedance-type devices due to their geared or otherwise nonlinear strong actuators. However, these devices are difficult to backdrive and require force sensors as inputs to control the device's position/velocity (admittance control [5]), or force (force control [2][6][7][8]). Although both structures have been implemented in HHUIs, each one has had to develop various methods for accounting for the associated shortcomings.

Despite the type of haptic structure, the literature has shown that only linear relationships have been applied between the virtual/robotic forces and the desired master forces. The issue with this widely used technique when it comes to HHUIs is that it does not optimally consider the characteristic differences between manipulation and grasping tasks. The research presented in [14] applied a nonlinear 
slave-master force profile relationship (Figure 1) to an impedance-type device to appropriately account for these differences based on the increased task complexity, task frequency, muscle dominance, user fatigue, the humans ability to sense just noticeable force differences, and the associated brain activity during manipulation tasks compared to grasping tasks. The profile can also be viewed from a resiliency aspect.

Taking such a vantage point it is understood that manipulation tasks require numerous degrees of freedom and slipping/rolling motions between the fingers and within the palm to accommodate and dexterously maneuver objects of various weight, shape, and size. This argues that the manipulation tasks are very resilient with respect to these object parameters, however, minor force perturbations beyond the anticipated gravitational influence can cause the object to significantly move in undesired ways. Alternatively, grasping tasks are much more resilient to unanticipated applied forces and transcend through a manipulation task to accommodate the object parameters.

As previously stated, generally HHUIs either provide sufficient force feedback to communicate the quantity and magnitude of the forces associated with either manipulation or grasping but not both. The devices that have been developed to complement grasping include [3] and the ones that have been developed to complement manipulation include [5]. In response to this limitation in the HHUI state-of-the-art, this research has developed an HHUI that has sufficient workspace, speed, and force to apply the developed nonlinear force profile and execute intuitive/transparent teleoperation manipulation and grasping tasks. The novelty associated with this research is that, unlike the linear relationship imposed in conventional systems which essentially treats manipulation and grasping tasks the same, the proposed system is tailored to the physical properties and resiliency of the hand in executing various tasks. Consequently, performance is enhanced and device size can be reduced for devices which the nonlinear profile is applied.

The proposed strategy can also be ubiquitously applied to any other haptic system (impedance-type or admittance-type) to appropriately relate the dynamic conditions of an environment with the human's ability to perceive the forces. By emphasizing the portions where human intuition is most impaired by conventional scaling and reducing emphasis on those portions where it is not as significant promises to correlate activities such as operating a backhoe to dig a trench, flying a plane, etc..

The remainder of this paper will present the nonlinear master-slave force profile complementing the hand's R1/R2 characteristics (Section II), the developed HHUI capabilities (Section III), the generated force controller (Section IV), experiment (Section V), results (Section VI), and conclusions (Section VII).

\section{Nonlinear Master-Slave ForCe Profile}

The applied nonlinear (NL) master-slave force profile is presented in Figure 1. In the profile there are two distinctive portions of the force profile which correspond to manipulation force execution levels and grasping force execution levels. The division between these regions occurs at approximately $30 \%$ of the user's Maximum Voluntary Force (MVF). In general it has been established that the fingers/thumb can exert a MVF of $50 \mathrm{~N} / 100 \mathrm{~N}$ respectively [11][12]. The manipulation region consists of no-load hand positioning, rolling/slipping of the object in the hand, and light grasping tasks with the user's fingertips and distal phalanges (referred to herein as Region 1 (R1)) while the remainder of the force space primarily consists of robust grasping (referred to herein as Region 2 (R2)). The figure also includes a linear $(\mathrm{L})$ profile that will be utilized in this study's experiments to represent the currently employed strategies.

In Figure 1, (L) exhibits a 1:2.5 force relationship between the user interface and robot throughout the force space, and (NL) exhibits a 1:1 relationship in R1 and a 1:7 in R2 with a smooth transition for the robotic forces between $11 \mathrm{~N}$ to $20 \mathrm{~N}$. The smooth transition was achieved using a third order polynomial that matches the location and slope of the R1 and $\mathrm{R} 2$ slopes at the ends of its $9 \mathrm{~N}$ span. The equations for this profile will be presented in the force control section below with particular emphasis on the variable in the force control algorithm in which it is applicable.

\section{DeVEloped Haptic Hand User InTERFACE CAPABILITIEs To COMPLEMENT NONLINEAR ForCE PROFILE}

The Haptic User Interface's Finger (HUIF) developed in this research is of similar kinematic form as that of most industrial elbow manipulators [18] and the HIROIII [2]. That is to say, it essentially has a two DOF universal joint at its base and a DOF revolute joint, which is parallel to one of the universal joint's DOF, between its two links. This 3 active DOF structure enables 3 dimensional force feedback at the fingertip connection points. The fingertip connection is a spherical magnetic joint like the HIROIII device which allows

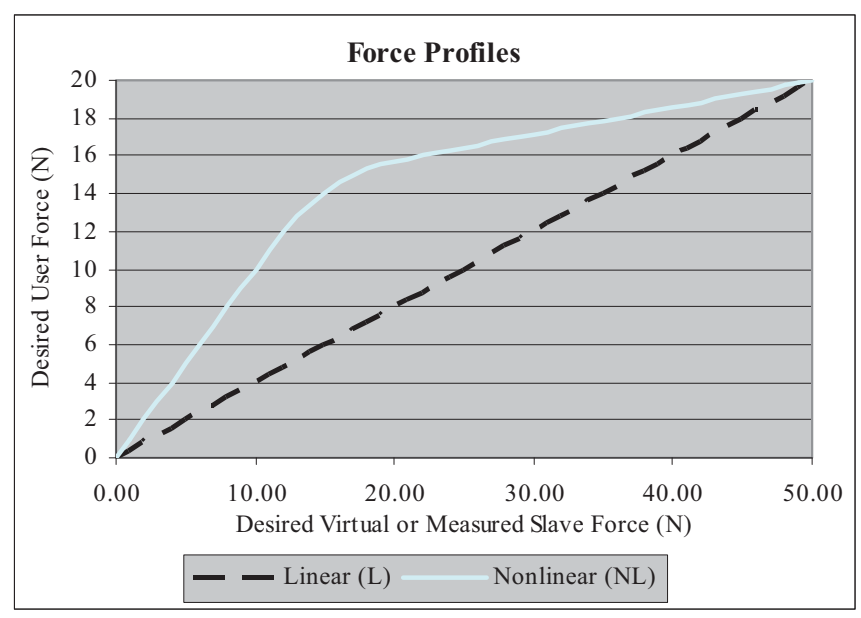

Figure 1. The force profiles evaluated in this study 
free motion of the remaining 3 DOF needed to accommodate the finger's orientational differences. The controlled forces (Fx, Fy, Fz) and free torques (Tx, Ty, Tz) can be seen in Figure 2. Due to proprietary considerations more particularities associated with the device will not shown here.

The developed HUIF is also an admittance-type of device and implements ATI's nano-17 force/torque sensors in its peripheral required for the employed force control. However, the HUIF developed by this research has considerably more fingertip force and speed generating capability than its closest HHUI counterpart (HIROIII), (23N vs. $3.6 \mathrm{~N}$ and $0.45 \mathrm{~m} / \mathrm{s}$ vs. $0.2 \mathrm{~m} / \mathrm{s}$ ). The enhanced capacities were integrated such that the device could move with the same speed capability as that of the human finger and accommodate the developed (NL) force profile.

The prototype is currently fabricated out of rapid prototyped components and controlled by a National Instrument real-time system using FPGA interface components. The elaborate hardware was implemented to ensure that the desired haptic $1 \mathrm{kHz}$ sampling rate was achieved even with all the necessary calculations performed during each iteration. If this proves to be too computationally burdensome in the future, with the inclusion of more HHUI fingers, the calculations will be divided into an outer loop (kinematic calculations) and inner loop (control calculations). Implementers of this technique have reported that the outer loop can run 5-10 times slower than the inner loop, with little sacrifice in performance [19].

\section{FORCE CONTROL}

The developed force controller will appropriately utilize the general n-link manipulator dynamic equation as shown in equation (2). In equation (2) $M(\theta)$ is the inertia matrix, $C(\theta, \dot{\theta}) \dot{\theta}$ is the Coriolis and centripetal torques, $V(\theta, \dot{\theta})$ is the friction (coulomb, viscous, etc.) related torques, $G(\theta)$ is the gravitational related torques, and $\tau$ is the control torque.

$$
M(\theta) \ddot{\theta}+C(\theta, \dot{\theta}) \dot{\theta}+G(\theta)+V(\theta, \dot{\theta})=\tau
$$

The force control system candidates considered for this system included the Nonlinear Proportional Derivative NPD technique [20], computed torque [21], and explicit force controllers [2][7][8]. These systems were generally developed to control a manipulator's downward force on a fixed surface which employs certain simplifications compared to the device presented in this research where force will be controlled in all three directions during free translation and interaction. Of these techniques, explicit force control was implemented at this juncture in the research.

The explicit force controller used for the admittance-type HIROIII device [2] utilizes gravitational compensation and active damping in their force PI control algorithm, but the author cannot locate the controller gains used by the device or if the means for selecting these gains have a model related basis.
The explicit force controller implemented by [7] utilizes a model based root locus approach in its comparison of various forms of conventional position, integral, and derivative force controllers. In order to achieve this end, the controller also utilizes active gravitational compensation as well as friction, Coriolis, and centripetal compensation in the development of its robotic arm model thus leaving the inertial terms for the controller gains to accommodate. Reference [7] also implemented active damping in the design as it provided a stabilizing effect and took advantage of some simplifications available due to its application on a fixed surface rather than a moving point. The results of the study showed that integral control generated the most effective performance.

The explicit force controller utilized in [8] also included the inertial term in addition to the terms compensated for by [7] and thus approximately compensating for all of the haptic device's dynamic effects, leaving control of just the force of interest. Issues associated with inappropriate approximations arise from this technique but appeared to be adequately compensated for by the integral term in its force PI controller.

This research implemented a proportional with feed forward force controller and gravity/friction compensation. The compensated friction term $(V(\theta, \dot{\theta}))$ was a combination of coulomb and viscous friction values extracted from the motor behavior as functions of the angular velocity [22]. The controller gains were selected to achieve the appropriate free motion bandwidth and virtual surface interaction performance without instability as described in the experiment section. The inertial and Coriolis/centripetal terms were not accounted for because the accelerations and velocities experienced during the manipulation task were not significant and it was assumed that the interaction force with the surface would dominate these influences thus making them negligible. A more elegant

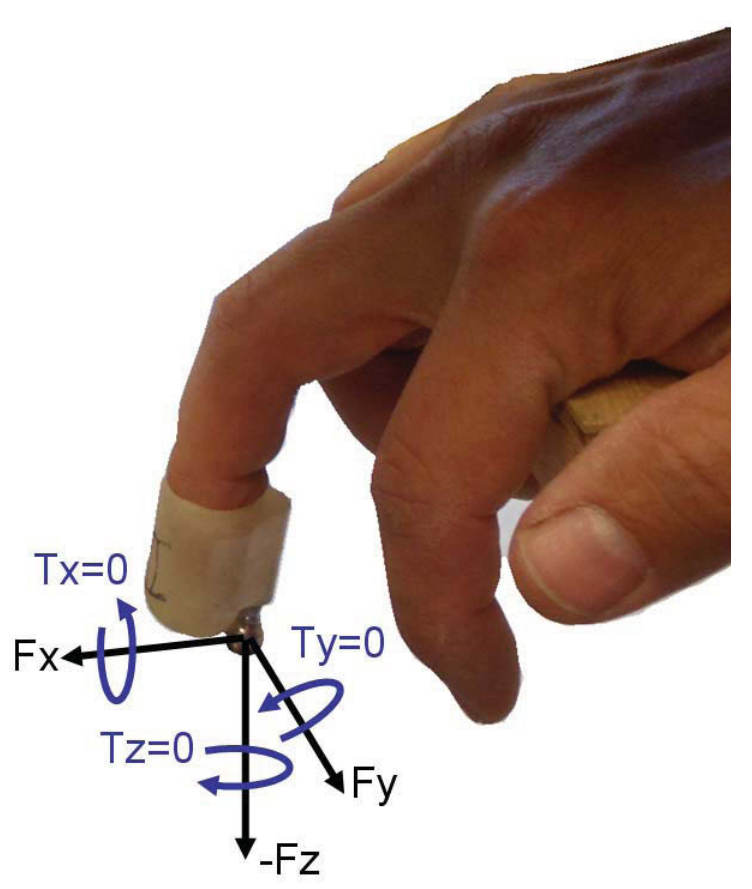

Figure 2: Force feedback provided back to user by haptic finger device 
consideration of these effects will be made in the next iteration of this research's controller. However, in this study both the (L) and (NL) profiles will be subjected to the same circumstances thus supporting the validity of this study's comparison.

\section{A. Control System Algorithm}

The control law currently employed in this system is shown in equation (3) with the $f_{e}$ term presented in (4) where $f_{m}$ is the measured sensor force and $f_{\text {des }_{L, N L}}$ are the linear and non-linear force profile correlations discussed above and presented in (5) - (8). The linear profile is expressed by (5) while the R1, transition from R1 to R2, and R2 segments of the nonlinear profile are expressed in (6) - (8) respectively where $f_{V R}$ is the calculated virtual or measured slave robot force.

$$
\begin{aligned}
& \tau=J^{T}\left(K_{1} f_{e}+f_{\operatorname{des}_{L, N L}}\right)-\hat{G}(\theta)-\hat{V}(\theta, \dot{\theta}) \\
& f_{e}=f_{d e s_{L, N L}}-f_{m} \\
& f_{\operatorname{des}_{L}}=0.4 f_{V R} \\
& f_{\text {des }_{L}}=f_{V R}
\end{aligned}
$$

$f_{\operatorname{des}_{N L R 12}}=0.00118 \cdot\left(f_{V R}\right)^{3}-0.1023 \cdot\left(f_{V R}\right)^{2}+2.823 \cdot\left(f_{V R}\right)-9$.

$$
f_{\text {des }_{N L R 2}}=(1 / 7) f_{V R}+(90 / 7)
$$

\section{EXPERIMENT/RESULTS}

This study implemented three experiments: one to select the system gains based on apparent device transparency via a finger tapping test (Figure 3), the second will analyze the performance difference between the two profiles given a manipulation-like task of matching a circle pattern on a surface (Figure 4), and the third is a grasping-type task on a collapsible cup model (Figure 5). At this point in the research only one subject has been utilized in the above experiments but multiple repetitions of the same experiments were required in order to achieve desirable statistical data and distributions.

\section{A. Transparency Study}

The transparency study consisted of the user performing 11 taps of sufficient displacement in the $\mathrm{x}, \mathrm{y}$, and $\mathrm{z}$ directions, independently. For each direction the proportional gain was increased from 1 to 100 in steps of 10 and the response time to complete the task was recorded. The experiment was performed by first implementing the (L) profile then the (NL) profile. The tapping action is commonly used in finger research as it assesses motor speed and control [23].

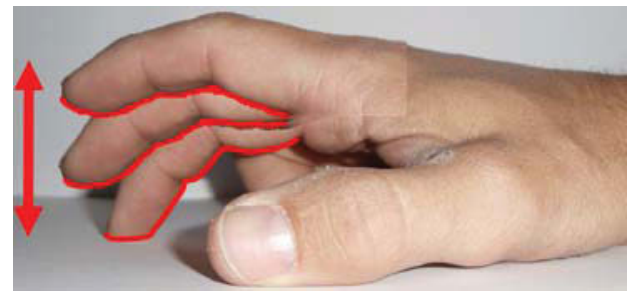

Figure 3. Finger exhibitting tapping motion

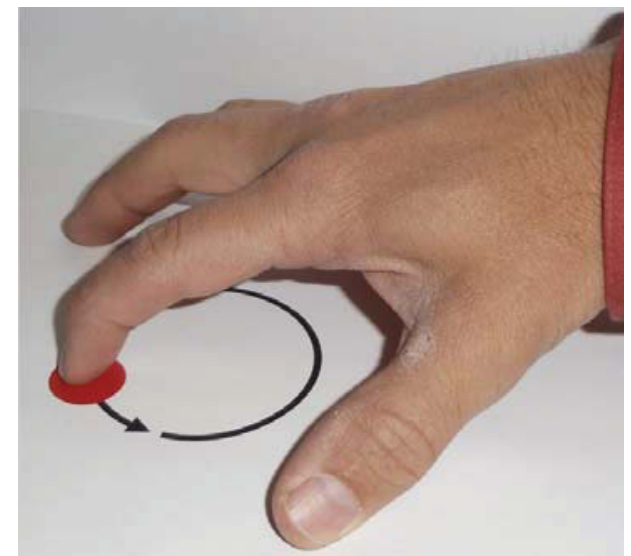

Figure 4. Finger demonstrating executed manipulation task

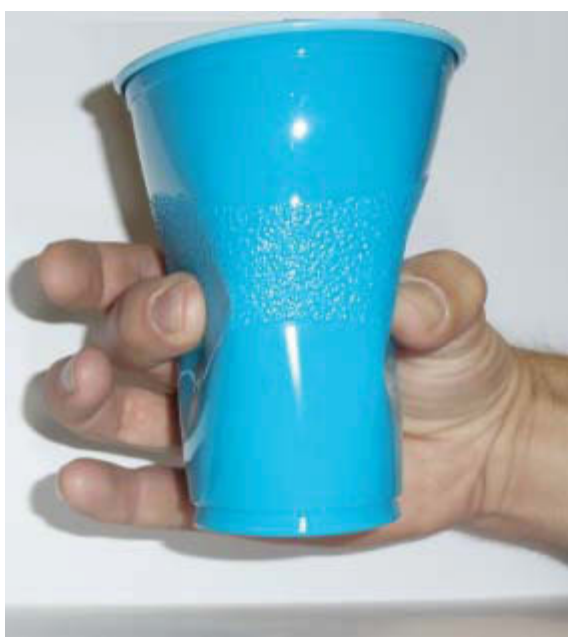

Figure 5. Fingers demonstrating simulated grasp task.

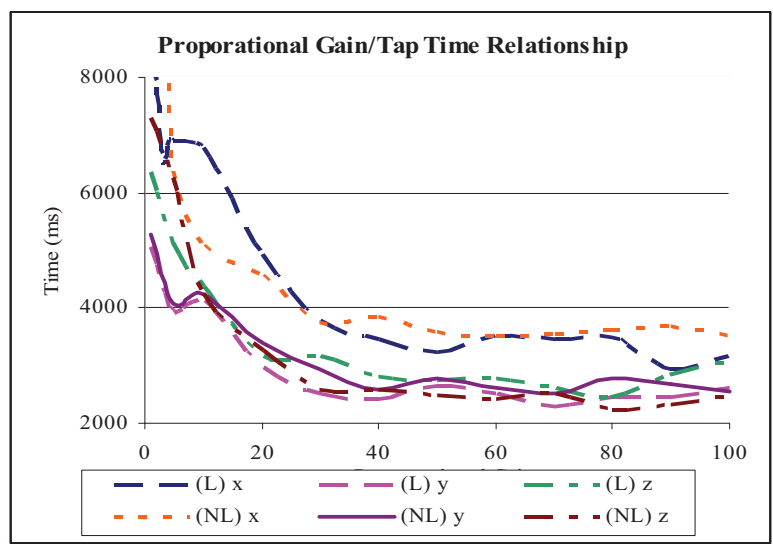

Figure 6. Proportional gain to time required to complete 11 taps graph. 
The resulting gain/time graphs for the 6 experiments are shown in Figure 3. This figure shows that all the time histories level out at a gain around 30 despite their correspondence to the $\mathrm{L}$ or NL profile. The resulting time values were also commensurate with the time required to complete the same task without being attached to the device. Therefore, a proportional gain of 30 was used in the following manipulation experiment.

\section{B. Manipulation Study}

The manipulation study consisted of the user attempting to match a circular pattern on a virtual surface with their fingertip for five complete rotations while also attempting to avoid the application of a virtual downward force in excess of $8 \mathrm{~N}$. The $8 \mathrm{~N}$ threshold was selected because it is near the middle of the finger's manipulation region at $16 \%$ of the finger's MVF and introduces a level of delicateness to the experiment.

The task was simulated with a black circle to represent the target path and a colored dot to represent the fingertip with the dot's color representing the state of the normal force applied (blue - zero force and no surface contact, green - force applied in a target range, red - force exceeding the target range). The experiment was performed by each profile three times on three frictionless virtual surfaces of different stiffness's $(1000 \mathrm{~N} / \mathrm{m}$, $750 \mathrm{~N} / \mathrm{m}$, and $500 \mathrm{~N} / \mathrm{m}$ ) resulting in 9 tests per profile. In order to reduce the computational data storage during the experiment, which resulted in undesired time delays, equations (10) and (11) were reduced to the iterative forms in (12) - (15) to calculate the average and standard deviation of the position error and force values at the current iteration $(N)$ given the current error/force value $\left(E_{N}\right)$, sum of the error/force $\left(T E_{N-1}\right)$, and sum of the error/force squared $\left(S E s q_{N-1}\right)$ from the previous iteration. The results from the experiment are embodied in Table I and Table II.

$$
\begin{aligned}
& \bar{E}=\left(\sum E\right) / N \\
& \sigma_{N}=\sqrt{\frac{\sum(E-\bar{E})^{2}}{N-1}}=\sqrt{\frac{\sum E^{2}-2\left(\sum E\right) \bar{E}+\bar{E}^{2}}{N-1}} \\
& \bar{E}_{N}=\frac{\bar{E}_{N-1} *(N-1)+E_{N}}{N} \\
& T E_{N}=T E_{N-1}+E_{N} \\
& S E s q_{N}=S E s q_{N-1}+E_{N}{ }^{2}
\end{aligned}
$$$$
\sigma_{N}=\sqrt{\frac{S E s q_{N}-2 * T E_{N} * \bar{E}_{N}+\bar{E}_{N}{ }^{2}}{N-1}}
$$

TABLE I. AVERAgE MANIPULATION STUdy RESUlts For EACH STIFFNESS AND PROFILE

\begin{tabular}{|c|c|c|c|c|c|c|c|c|c|}
\hline Profile & $\begin{array}{c}k \\
(\mathrm{~N} / \mathrm{m})\end{array}$ & $\begin{array}{c}T \\
(\mathrm{~s})\end{array}$ & $\begin{array}{c}\text { re avg } \\
(\mathrm{mm})\end{array}$ & $\begin{array}{c}\text { re std } \\
(\mathrm{mm})\end{array}$ & $\begin{array}{c}F z \text { avg } \\
(\mathrm{N})\end{array}$ & $\begin{array}{c}F z \text { std } \\
(\mathrm{N})\end{array}$ & $\begin{array}{c}\text { up } \\
\%\end{array}$ & $\begin{array}{c}\text { down } \\
\%\end{array}$ & $\begin{array}{c}\text { mid } \\
\%\end{array}$ \\
\hline $\mathrm{L}$ & 1000 & 6.7 & -1.8 & 3.5 & -7.21 & 3.57 & 3 & 42 & 55 \\
\hline $\mathrm{NL}$ & 1000 & 6.5 & -2.4 & 2.7 & -4.70 & 3.26 & 9 & 25 & 67 \\
\hline $\mathrm{L}$ & 750 & 7.7 & -1.9 & 2.3 & -5.84 & 2.56 & 0 & 26 & 74 \\
\hline $\mathrm{NL}$ & 750 & 6.1 & -2.2 & 2.3 & -3.83 & 2.65 & 7 & 3 & 90 \\
\hline $\mathrm{L}$ & 500 & 8.1 & -1.5 & 3.2 & -6.29 & 2.56 & 4 & 30 & 66 \\
\hline $\mathrm{NL}$ & 500 & 7.9 & -2.4 & 2.5 & -3.76 & 1.94 & 4 & 0 & 96 \\
\hline
\end{tabular}

TABLE II. Total Average Manipulation Study Results For Each PROFILE

\begin{tabular}{|c|c|c|c|c|c|c|c|c|c|}
\hline Profile & $\begin{array}{c}k \\
(\mathrm{~N} / \mathrm{m})\end{array}$ & $\begin{array}{c}T \\
(\mathrm{~s})\end{array}$ & $\begin{array}{c}\text { re avg } \\
(\mathrm{mm})\end{array}$ & $\begin{array}{c}\text { re std } \\
(\mathrm{mm})\end{array}$ & $\begin{array}{c}\text { Fz avg } \\
(\mathrm{N})\end{array}$ & $\begin{array}{c}\text { Fz std } \\
(\mathrm{N})\end{array}$ & $\begin{array}{c}\text { up } \\
\%\end{array}$ & $\begin{array}{c}\text { down } \\
\%\end{array}$ & $\begin{array}{c}\text { mid } \\
\%\end{array}$ \\
\hline $\mathrm{L}$ & - & 7.5 & -1.7 & 3.0 & -6.3 & 2.9 & 2 & 32 & 65 \\
\hline $\mathrm{NL}$ & - & 6.8 & -2.4 & 2.5 & -4.1 & 2.6 & 6 & 9 & 85 \\
\hline
\end{tabular}

Table I shows that the finger's ability to follow the circle for each given stiffness was relatively consistent. This is shown in that the average radius values in combination with their associated standard deviation exhibit significant overlap. However, such consistency was not exhibited with regard to the applied downward force. In fact the $\mathrm{L}$ profile executed forces that were $50-60 \%$ larger than that of the NL profile for each stiffness and exceeded the $8 \mathrm{~N}$ threshold 3 times as much as that of the NL profile. The user also reported significantly less concentration needed to execute the task with the NL profile than with the L profile.

Had equivalent concentration been executed for both profiles the force standard deviations would most likely have exhibited a similar trend as that of the corresponding average applied forces. However, because the force standard deviations are consistent testifies that the user did not monitor force variations as stringently when implementing the NL profile versus the $\mathrm{L}$ profile and was still able to produce better performance.

\section{Grasping Study}

The grasping study presented in this paper is consistent in setup and parameter as that performed in [14] using the Novint Falcon impedance-type haptic device. The grasp task consists of a simulation of a finger holding a plastic cup as it is filled with fluid. Although this grasp requires the thumb and palm in addition to the finger to hold the plastic cup, only the finger will be modeled here assuming that the other two constraints are displaced equally around the cup and act identically to that of the finger. The first factor that varies during the process of holding the cup stable while being filled includes the ability to sense the increase in weight. The hand grasp force must increase to retain the increasing weight but must also be aware of the fragile nature of the plastic cup which may collapse if held too firm. The second factor that varies is the stiffness of 
the cup due to the increasing pressure of the water against the walls. The equation used to generate the stiffness profile for quantifying these factors is presented in (15), (16), and (17).

$$
\begin{aligned}
& \vec{F}_{V R F_{-} \text {norm }}=k \cdot t \cdot\left(-\Delta x^{2}+b \cdot \Delta x\right)_{-} \underline{I F}-\left\langle r_{\text {finger }}<r_{\text {cup }}\right\rangle \\
& k=\left(4 \cdot F_{\max }\right) /\left(t_{\max } \cdot b^{2}\right) \\
& F_{w \max }=F_{\max } \cdot \mu
\end{aligned}
$$

In equation (15) the $b$ variable controls the width of the region between initial contact and cup collapse. The influence of the $b$ variable is that larger values provide the user with more available displacement and force range that they can work with to remain in the target region and thus also reduces the sensitivity to slight changes. The $k$ variable controls the height of the compliance profile to match the minimum calculated force threshold $\left(F_{\text {wmax }}\right)$ where the cup would slip through the user's fingers. The simulation provided a small vibration feedback to the user if the $F_{w \max }$ threshold was approached.

Each experiment was designed to last 10 seconds from initial virtual contact as the weight of the cup was linearly increased from 0 to an end target force $(35 \mathrm{~N})$. The $35 \mathrm{~N}$ value was calculated by multiplying the maximum desired normal force times $(50 \mathrm{~N})$ the coefficient of friction $(0.7)$. The maximum desired normal virtual force was selected such that they would transcend the entire horizontal axis of the force profiles embodied in Figure 1 and convert them into the corresponding desired user force. Each experiment would consist of five repetitions with the (L) profile and an equal number of repetitions with the (NL) profile. The user was asked to close their eyes during the experiment such that any visual feedback would be eliminated thus isolating the force feedback response.

Representative resulting profiles for the $\mathrm{L}$ and NL profiles are shown in Figure 7 and Figure 8 where the upper smooth line represents the maximum force the cup's compliance would allow the user to apply and the lower smooth line represents the minimum force that user is able to apply prior to cup slip. A vibration was added to the signal if the user dropped below the minimum force to communicate this behavior to the blinded user. As shown in the figures the general trend of the experiment was that the NL profile was slightly less erratic during initial contact and generally was better able to sense the force feedback needed to control the cup without slippage throughout the entire profile.

Quantitative values associated with the grasp study are contained in Table III. The results indicate how well the user stayed above the minimum force line associated with cup slip and generally were within the minimum and maximum force limits. By staying within these boundaries indicates how well the user retained control of the virtual cup for Region 1 (R1), the transition between Region 1 and 2 (R12), and Region 2
(R2). The first parameter for each region was calculated by determining what percentage of the time the user fell below the threshold. The second parameter for each region was initially going to be calculated by determining the average force error between the applied force and the midline force (taken as the average of the maximum and minimum curves). However, because the curves are so dramatically different it was decided that describing this as a percentage would be most applicable in order to adequately compare the two results

\begin{tabular}{|c|c|c|c|c|c|c|}
\hline & $\begin{array}{c}\text { Below } \\
\text { Limit R1 } \\
\text { (\%) }\end{array}$ & $\begin{array}{c}\text { Relative } \\
\text { Range R1 } \\
\text { (\%) }\end{array}$ & $\begin{array}{c}\text { Below } \\
\text { Limit R12 } \\
(\%)\end{array}$ & $\begin{array}{c}\text { Relative } \\
\text { Range } 12 \\
\text { (\%) }\end{array}$ & $\begin{array}{c}\text { Below } \\
\text { Limit R2 } \\
\text { (\%) }\end{array}$ & $\begin{array}{c}\text { Relative } \\
\text { Range R2 } \\
\text { (\%) }\end{array}$ \\
\hline$L$ & 38 & -200 & 6 & -47 & 34 & -74 \\
\hline $\mathrm{NL}$ & 33 & -164 & 7 & -64 & 15 & -56 \\
\hline
\end{tabular}
(e.g. $-50 \%$ corresponds to the average applied force lying half way between the midline and the minimum force curve).

The results of Table III indicate that for R1 and R12 both profiles performed approximately the same and although in R2 the NL profile did not drop below the force limit as much as the $\mathrm{L}$ profile it is not to a very dramatic degree. Thus it can be said that the NL profile performed just as well as that of the $\mathrm{L}$ profile with respect to the grasping task experiment shown

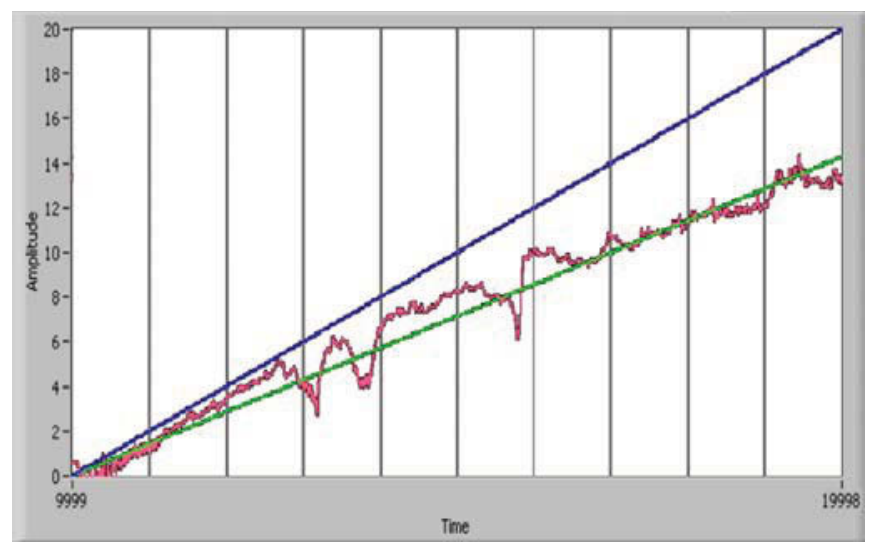

Figure 7. Representative L profile erradic and saturation behavior

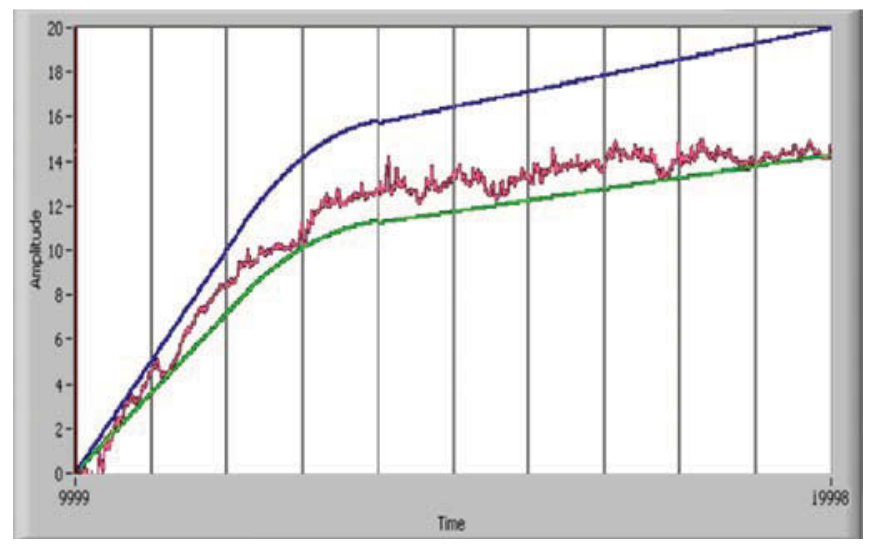

Figure 8. Representative NL profile erradic and saturation behavior 
here even though its reduced sensitivity provided in R2 further demonstrating the hand's resiliency to varying degrees of force changes once force closure is achieved.

Additionally, the subject did report slightly more fatigue after performing the NL experiments than the L experiments. However, the subject also reported that the NL profile required less concentration during the task than the L profile.

\section{CONCLUSION AND FUtURE WORK}

This study has demonstrated that a force profile that appropriately complements the human hand's resiliency provides significant performance improvements over the conventional linear force profile when applied to a haptic finger user interface for BOTH manipulation and grasping tasks. Although the results are specific to a haptic finger the force profile correlation technique has significant potential in enhancing the transparency between humans and machines by emphasizing/correlating human physical/mental intuition factors with a device's mechatronic features. As a consequence, user performance with such devices can be greatly enhanced while size can be reduced.

Particular to this study, it was demonstrated that the developed device has sufficient bandwidth to offer the user transparent free motion operation given gains greater than 30 . The manipulation study showed that both profiles produce commensurate circle following performance but the NL profile demonstrated superior downward force management capabilities during the operation. The grasp study demonstrated that the NL profile provided slightly better performance than the $\mathrm{L}$ profile which is an acceptable outcome considering that our hypothesis that the finger is more resilient to force variations during grasping task and the reduced sensitivity provided by the NL in R2 does not adversely impact performance.

Future work will consist of implementing a more elegant gain/stability analysis in the development of a force controller using an adaptive root locus gain selection strategy or nonlinear control strategy to enhance controller performance in the presence of the nonlinear force profile relationship. The primary challenge in the proposed controller is not that there is nonlinearity in the system but that the nonlinearity occurs in the force error term which is not a convention formulation and opens up novel research areas. Additionally, the nonlinear profile may be suitably modified to account for the reported fatigue phenomena, and a more mathematical means of generating the force profile for each individual user to enhance intuition may be developed.

A more advanced friction compensation scheme is also intended to enhance some of the observed behaviors of the mechanisms. The research will account for additional variables such as torque in addition to the current velocity input.

\section{AKNOWLEDGEMENT}

Prepared for the U.S. Department of Energy through the INL LDRD Program Under DOE Idaho Operations Office Contract DE-AC07-05ID14517.

\section{REFERENCES}

[1] Goertz, R. C., "Remote-Control Manipulator," Patent 2,632,574, December 1949.

[2] Endo, T., et. al., "Five-Fingered Haptic Interface Robot: HIRO III," IEEE Transactions on Haptics, Vol. 4, No. 1, January-March 2011.

[3] CyberGlove Systems LLC, "CyberGrasp ${ }^{\text {TM }}$ System User Guide v2.0," (2009).

[4] Avizzano, C., Bargagli, F., Frisoli, A.,Bergamasco, M., "The hand force feedback: analysis and control of a haptic device for the human-hand," Proceedings of IEEE International Conference on Systems, Man, and Cybernetics, vol. 2, pp. 989-994. Nashville, TN (2000).

[5] Abbott, J., Okamura, A., "Pseudo-Admittance Bilateral Telemanipulation with Guidance Virtual Fixtures," The International Journal of Robotics Research, Vol. 26, No. 8, August 2007.

[6] An, C., Hollerbach, J., "Dynamic Stability Issues in Force Control of Manipulators," Vol. 4, Pages 890-896, 1987.

[7] Volpe, R., Khosla, P., "A Theoretical and Experimental Investigation of Explicit Force Control Strategies for Manipulators," IEEE Transactions on Automatic Control, Vol. 38, No. 11, November 1993.

[8] Carignan, C., Cleary K., "Closed-Loop Force Control for Haptic Simulation of Virtual Environments," Haptics-e, Vol. 1, No.2, February 23, 2000.

[9] Hogan, N., "Impedance Control: An Approach to Manipulation: Part III - Applications," Journal of Dynamic Systems, Measurement, and Control, Vol. 107/17, March 1985.

[10] H. K. Khalil, Nonlinear Systems, $3^{\text {rd }}$ edition, Prentice Hall, Upper Saddle River, New Jersey, 2002.

[11] Astin, Angela DiDomenico, "Finger force capability: measurement and prediction using anthropometric and myoelectric measures," Thesis, Virginia Polytechnic Institute and State University, 1999.

[12] Boatright, J. R. et. al., "Measurement of Thumb Abduction Strength: Normative Data and a Comparision With Grip and Pinch Strength," The Journal of Hand Surgery / Vol. 22A No. 5 September 1997.

[13] Tan, H.Z., et. al, "Human Factors for the Design of Force-Reflecting Haptic Interfaces", Dynamic Systems and Control, DSC-Vol.55-1, 1994.

[14] Crawford, A., "Nonlinear Force Profile Used to Increase the Performance of a Haptic User Interface for Teleoperating a Robotic Hand," Proceedings of NPIC \& HMIT, San Diego, CA, 2012.

[15] Zheng, J.Z., Rosa, S., Dollar, A.M., "An Investigation of Grasp Type and Frequency in Daily Household and Machine Shop Tasks," Proceedings of IEEE International Conference on Robotics and Automation, Shanghai, China, May 9-13, pp.4169-4175 (2001).

[16] Ehrsson, H.,H., et. al, "Cortical Activity in Precision- Versus PowerGrip Tasks: An fMRI Study", The Journal of Neurophysiology, 83:528536, 2000.

[17] Kutch, J. J., Valero-Cuevas, F. J., "All muscles are redundant but some are less redundant than others," poster presentation for USC Viterbi School of Engineering Brain-Body Dynamics Lab.

[18] Lloyd, J., Hayward, V., "Kinematics of Common Industrial Robots," Robotics and Autonomous Systems, Vol. 4, Issue 2, pages 169-191, June 1988.

[19] Maples, J., Becker, J., "Experiments in force control of robotic manipulators," Proceedings of the IEEE Conference on Robotics and Automation, Pages 695-702, 1986.

[20] Xu, Y., Hollerbach, J.M., and Ma, D., "A nonlinear PD controller for force and contact transient control," IEEE Control Systems, 15, no. 1, 1995, pp. 15-21.

[21] J. J. Craig, Introduction to Robotics: Mechanics and Control, 3rd edition, Prentice Hall,Upper Saddle River, New Jersey, 2005.

[22] Canudas, C., Astrom, K. J., Brausn, K., "Adaptive Friction Compensation in DC-Motor Drives," IEEE J. Robotics and Automation, RA-3, No. 6, Pages 681-685, Dec. 1987.

[23] Christianson, M., Leathem, J., "Development and Standardisation of the Computerised Finger Tapping Test: Comparison with Other Finger Tapping Instruments," New Zealand Journal of Psychology, Vol. 33, 2004. 